\title{
Diabetes Patients Perceptions on Drugs Consultation Program by Pharmacists
}

\author{
Budi Sumaryono $^{1}$, Moelyono Moektiwardoyo ${ }^{2}$, Ari Yulianto ${ }^{1}$ \\ ${ }^{1}$ Indonesian Air Force Pharmaceutical Institution, Bandung, West Java, Indonesia \\ ${ }^{2}$ Faculty of Pharmacy, Universitas Padjadjaran, Bandung, West Java, Indonesia
}

\begin{abstract}
Drugs consultation by pharmacist is an important service to improve the quality of medication use in patients with type 2 diabetes mellitus (T2DM). Perception of patients regarding this service is very useful to boost pharmacists confidence to conduct such service. The purpose of this study is to provide an overview regarding interests and categories of drugs consultation needed by T2DM patients and to determine the effect of quality of drugs consultation on patients interest to participate in such program. Survey and interviews were conducted with T2DM patients in outpatient clinic, Dr. M. Salamun Air Force Hospital, Bandung during April-June 2013. One hundred patients were recruited for this study. Most of the patients consumed combination of oral antidiabetic and non-antidiabetic medication $(71 \%)$ and were given polypharmacy (45\%). The results showed that the information regarding drugs interactions, adverse drugs reaction (ADR), and drugs dosage were among the aspects that should be included in drugs consultation service by pharmacists. The quality of drug information service was associated with increased interest to participate in such program. These aspects should be considered in the implementation of drugs consultation program by pharmacists.
\end{abstract}

Keywords: drugs consultation, T2DM, pharmacists

\section{Introduction}

The prevalence of type 2 diabetes mellitus (T2DM) has increased dramatically. Each year, 7 million people are diagnosed with T2DM and every 10 seconds a person dies due to this disease. ${ }^{1}$ T2DM patients are tend to have polypharmacy in long period of time which may increase the risks of experiencing problems related to medication use. Such problems potentially have an impact on desired health outcomes. ${ }^{2}$
Pharmacists are in the excellent position to tackle the problem related to medication use. Drug consultation is one of the important services by pharmacists to improve the quality of drug use in patients. ${ }^{3}$ Drug consultations conducted for patients with chronic diseases T2DM are useful to increase adherence to medication, enhance drug safety, and optimize health outcomes. ${ }^{4,5}$ 
Monitoring of the medication use by pharmacists was not yet performed in Dr. M. Salamun Air Force Hospital Bandung. Although the regulation states that the pharmacist has the responsibility and competence to conduct drugs monitoring and consultation to the patients, most pharmacists hesitate to perform such service. In the midst of confidence issues, understanding the patients perception on drugs consulatation program can help to increase the confidence of the the pharmacists to conduct such program. ${ }^{6}$

Perceptions of an existing pharmaceutical service are presumed as the representation of the patient interest to participate in drugs consultation program by pharmacists. With the understanding of the perception of patients on this service, pharmacists can determine the right programs and activities in developing drug consultation program that are needed by the patients. Therefore, this study was conducted to investigate patients perception regarding drugs consultation service by pharmacists. ${ }^{7}$

\section{Methods}

This research was an observational study with descriptive and analytic survey method. The variables studied in this research were the quality of drug information and patient interest for drug consultation program.

This study was conducted from July to September 2013 at Dr. M. Salamun Air Force Hospital, Bandung. Patient sampling was determined by Slovin formula. Considering the number of T2DM patients at study location and estimation of the outcomes, 100

Table 1. Characteristics of participants

\begin{tabular}{lc}
\hline \multicolumn{1}{c}{ Characteristics } & $\boldsymbol{n}$ (percentage) \\
\hline Sex & \\
Male & $45(45)$ \\
Female & $55(55)$ \\
Age & \\
50-54 years old & $29(29)$ \\
$55-59$ years old & $34(34)$ \\
60-64 years old & $37(37)$ \\
Level of education & \\
Elementary school & $10(10)$ \\
Junior high school & $23(23)$ \\
Senior high school & $31(31)$ \\
College & $36(36)$ \\
Pharmacotherapy & \\
Single oral antidiabetic drug & $4(4)$ \\
Combination of anti-diabetics drugs & $25(25)$ \\
Combination of anti-diabetics and & $71(71)$ \\
non-diabetics drugs & \\
\hline
\end{tabular}


patients needed to conduct this study. The inclusion criteria in this study were T2DM patients who received outpatient pharmacy service during April-June 2013; were able to communicate well, and aged 45 to 65 years old. We excluded patients with mental disorder.

Data was collected through Likert-scale questionnaires and interview. Data collected included patient characteristics, patient perceptions of drug information quality and patient interest in drug consultation by pharmacist. Information needed during drugs consultation service were classified as adverse drug reactions, drugs dosage and administration, drugs interaction, efficacy and indication of drugs, characteristics and identification of drugs, type of medication available, and how to store the drugs. ${ }^{7}$
The questionnaire consisted of 13 indicators about the quality of drug information and patients interest to participate in drug consultation by pharmacists. Prior to this test, the validity and reliability test were conducted on 30 outpatients. Testing was conducted using partial least square (PLS). The results showed that all of the statements were valid and reliable. The analytical tool to verifiy the analysis was structural equation modeling (SEM) using PLS.

\section{Results and Discussion}

\section{Validity and reliability}

All indicators of drug consultation interest variables had a significant loading factor. The value of composite reliability was showed to be reliable if the value is above 0.7 . The composite reliability of the questionnaire was 0.935 or reliable.

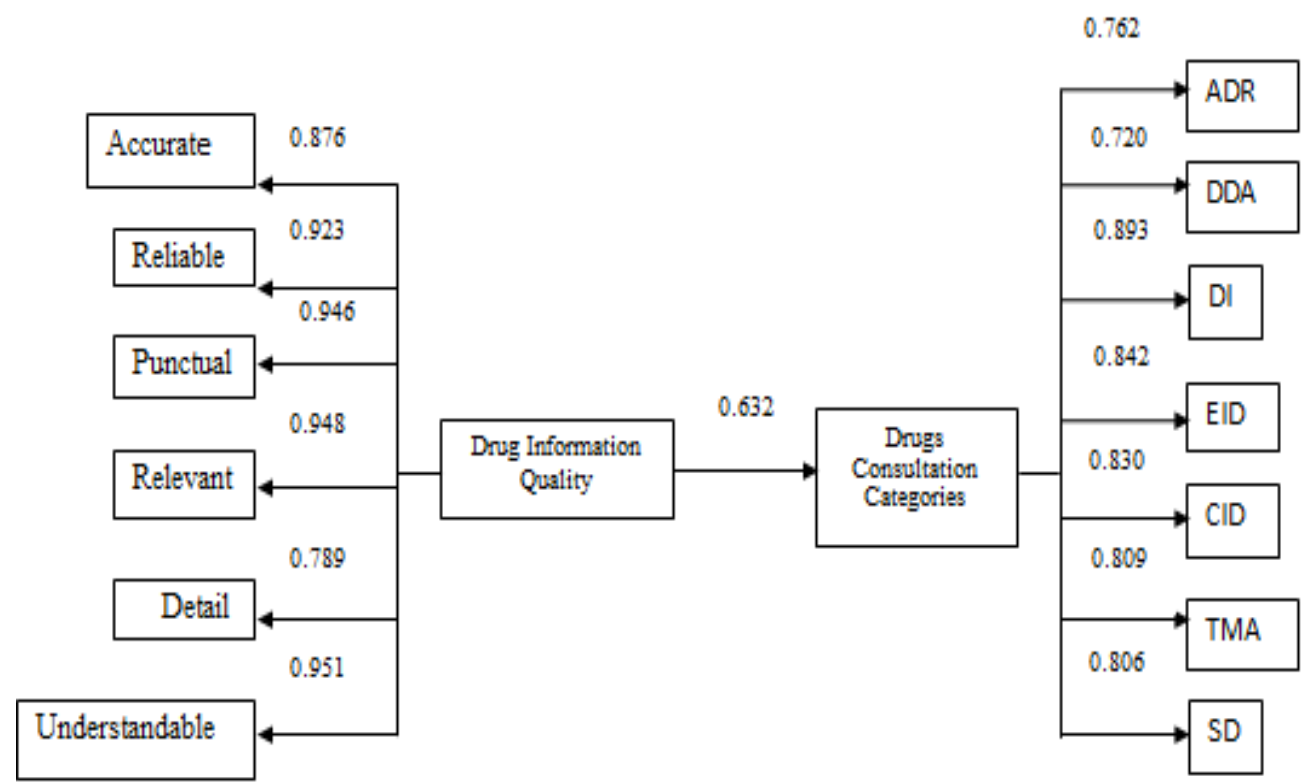

Figure 1. Component of drugs information quality and categories of drugs consultation preferred by T2DM patiens

\footnotetext{
ADR : Information regarding adverse drug reactions

DDA : Information regarding dosage and drug administration

DI : Information regarding drug interactions

EID : Information regarding efficacy and indication of drugs

CID : Information regarding characteristics and identification of drugs

TM : Information regarding type of medication available

SD : Information regarding how to store drugs
} 


\section{Characteristics of patients}

The majority of the patients were woman $(55 \%)$. Most patients were in the age category of 60-64 years old (36\%). Most patients had college education (37\%). Most patients used combination of antidiabetic and nonantidiabetic drug combinations (71\%). Most patients used 3-4 medications (45\%) (Table $1)$.

Age is one of the factors that can alter body metabolism. In order to have comparable characteristics of participants, we selected patients in the age group $>50$ years old. We found out many patients had good education. High level of education could lead to more critical attitude towards medication and health services they received. Combination of antidiabetics used in the patients included metformin with sulfonylureas, glinides, glucosidase inhibitors, thiazolidiones, DPP-4 inhibitor, and GLP-1 agonists. ${ }^{8}$

\section{Drugs consultation}

The results showed that the average score of patients perceptions regarding drug information quality and the interest to attend drugs consultation by pharmacists were $5.59 / 7.00$ and 5.17/7.00, respectively (Table 2 and 3). Both were in the good category. SEM analysis with PLSD was used to examine the effect of the quality of drug information to the drug consultation interest. The result of PLS analysis was obtained in a structural model in Figure 1.We found out that quality of drugs consultation significantly influenced the interest to participate in such program.

Information related to ADR, drugs interaction, and drug dosage were among the information preferred by the patients in drugs consultation program. The combination of anti-diabetic drugs could induce ADR such as hypoglycemia, gastrointestinal isorder, and weight gain. ${ }^{10,11}$ Beside, information related to the drug interaction was also needed by the patients due to the high prevalent of the use of concomitant medications related to other comorbities, such as antihypertensive, cardiovascular drugs, and renal drugs.

Research analysis model showed that the provision of good quality drug information service could influence the interest

Table 2. Patient perception on the quality of drug information

\begin{tabular}{|c|c|c|c|c|c|c|c|c|c|c|c|c|}
\hline \multirow{2}{*}{ Score } & \multicolumn{2}{|c|}{$\underset{\text { (accurate) }}{\mathbf{X}-\mathbf{1}}$} & \multicolumn{2}{|c|}{$\underset{\text { (reliable) }}{\mathbf{X}-2}$} & \multicolumn{2}{|c|}{$\begin{array}{c}\mathbf{X}-2 \\
\text { (punctual) }\end{array}$} & \multicolumn{2}{|c|}{$\underset{\text { (relevant) }}{\mathbf{X}-3}$} & \multicolumn{2}{|c|}{$\underset{\text { (detail) }}{\mathbf{X}-4}$} & \multicolumn{2}{|c|}{$\begin{array}{c}\mathbf{X}-\mathbf{5} \\
\text { (understandable) }\end{array}$} \\
\hline & $f$ & $x i$ & $f$ & $x i$ & $f$ & $x i$ & $f$ & $x i$ & $f$ & $x i$ & f & $x i$ \\
\hline 1 & 6 & 6 & 4 & 4 & 6 & 6 & 4 & 4 & 5 & 5 & 4 & 4 \\
\hline 2 & 2 & 4 & 3 & 6 & 7 & 14 & 4 & 8 & 7 & 14 & 1 & 2 \\
\hline 3 & 1 & 3 & 1 & 3 & 7 & 21 & 1 & 3 & 2 & 6 & 1 & 3 \\
\hline 4 & 10 & 40 & 5 & 20 & 12 & 48 & 10 & 40 & 9 & 36 & 9 & 36 \\
\hline 5 & 15 & 75 & 14 & 70 & 15 & 75 & 16 & 80 & 14 & 70 & 11 & 55 \\
\hline 6 & 28 & 168 & 30 & 180 & 25 & 150 & 23 & 138 & 26 & 156 & 35 & 210 \\
\hline 7 & 38 & 266 & 43 & 301 & 28 & 196 & 42 & 294 & 37 & 259 & 39 & 273 \\
\hline Amount & 100 & 562 & 100 & 584 & 100 & 510 & 100 & 567 & 100 & 546 & 100 & 583 \\
\hline $\begin{array}{c}\text { Score per } \\
\text { indicator }\end{array}$ & \multicolumn{2}{|c|}{5.62} & \multicolumn{2}{|c|}{5.84} & \multicolumn{2}{|c|}{5.1} & \multicolumn{2}{|c|}{5.67} & \multicolumn{2}{|c|}{5.46} & \multicolumn{2}{|c|}{5.83} \\
\hline Mean & \multicolumn{12}{|c|}{5.59} \\
\hline
\end{tabular}


Table 3. Patients preference on the category of drugs consultation service

\begin{tabular}{|c|c|c|c|c|c|c|c|c|c|c|c|c|c|c|}
\hline \multirow{2}{*}{ Score } & \multicolumn{2}{|c|}{ ADR } & \multicolumn{2}{|c|}{ DDA } & \multicolumn{2}{|c|}{ DI } & \multicolumn{2}{|c|}{ EID } & \multicolumn{2}{|c|}{ CID } & \multicolumn{2}{|c|}{ TM } & \multicolumn{2}{|l|}{ SD } \\
\hline & $\mathbf{f}$ & $x i$ & f & $x i$ & f & $x i$ & f & $x i$ & f & $x i$ & f & $x i$ & $\mathbf{f}$ & xi \\
\hline 1 & 10 & 10 & 4 & 4 & 8 & 8 & 12 & 12 & 25 & 25 & 20 & 20 & 26 & 26 \\
\hline 2 & 0 & 0 & 3 & 6 & 0 & 0 & 1 & 2 & 5 & 10 & 2 & 4 & 4 & 8 \\
\hline 3 & 0 & 0 & 2 & 6 & 1 & 3 & 0 & 0 & 0 & 0 & 0 & 0 & 0 & 0 \\
\hline 4 & 16 & 64 & 8 & 32 & 1 & 4 & 19 & 76 & 19 & 76 & 25 & 100 & 13 & 52 \\
\hline 5 & 11 & 55 & 11 & 55 & 14 & 70 & 12 & 60 & 15 & 75 & 12 & 60 & 12 & 60 \\
\hline 6 & 9 & 54 & 14 & 84 & 12 & 72 & 16 & 96 & 5 & 30 & 12 & 72 & 16 & 96 \\
\hline 7 & 54 & 378 & 58 & 406 & 64 & 448 & 40 & 280 & 31 & 217 & 29 & 203 & 29 & 203 \\
\hline Amount & 100 & 561 & 100 & 593 & 100 & 605 & 100 & 526 & 100 & 433 & 100 & 459 & 100 & 445 \\
\hline $\begin{array}{c}\text { Score } \\
\text { per indi- } \\
\text { cator }\end{array}$ & & & & & & & & 26 & & & & & 4.45 & \\
\hline Mean & & & & & & & & & & & & & & \\
\hline
\end{tabular}

to participate in drugs consultationby pharmacists, with the parameter coefficient of drug information on drug consultation interest was 0.632 with the t-value of 3.719 .

Initial information related to the drug use should be given by pharmacists themselves to build the trust regarding the roles and capabilities of pharmacists. Due to the high number of patients, pharmacists can prioritize the service to be given to patients with chronic disease, particularly T2DM. Individual and group counseling method can be used. Various tools can be used to enhance quality of pharmaceutical service such as the provision of brochure related to the drugs use and text message service reminder from pharmacists. ${ }^{12}$ The improvement in the quality of drugs information service could stimulate patients interest to consult regarding medication use to pharmacists.

\section{Conclusion}

The quality of drug information service was associated with the increased interest to participate in drugs consultation program by pharmacists. The information regarding drugs interactions, adverse drugs reaction (ADR), and drugs dosage were among the aspects that should be included in drugs consultation service by pharmacists.

\section{References}

1. Khan Mohammed A, Medarametla C, Rabbani MME, et al. Role of a clinical pharmacist in managing diabetic nephropathy: an approach of pharmaceutical care plan. Journal of Diabetes and Metabolic Disorders. 2015; 14:82.

2. Kocarnik BM, Liu CF, Wong ES. Does the presence of a pharmacist in primary care clinics improve diabetes medication adherence?. BMC Health Services Research. 2012;12:391.

3. Forouhi NG, Wareham NJ. Epidemiology of diabetes. Medicine. 2014;42(12):698702 .

4. Zaman Huri H, Chai Ling L. Drug-related problems in type 2 diabetes mellitus patients with dyslipidemia. BMC Public Health. 2013;13:1192.

5. Venkatesan R, Devi ASM, Parasuraman $\mathrm{S}$, et al. Role of community pharmacists 
in improving knowledge and glycemic control of type 2 diabetes. Perspectives in Clinical Research. 2012;3(1):26-31.

6. Mourão AOM, Ferreira WR, Martins MAP, et al. Pharmaceutical care program for type 2 diabetes patients in Brazil: a randomised controlled trial. International Journal of Clinical Pharmacy. 2013;35(1):79-86.

7. Salter SM, Karia A, Sanfilippo FM, et al. Effectiveness of e-learning in pharmacy education. American Journal of Pharmaceutical Education. 2014;78(4):83.

8. May M, Schindler C. Clinically and pharmacologically relevant interactions of antidiabetic drugs. Therapeutic Advances in Endocrinology and Metabolism. 2016;7(2):69-83.
9. Zolfaghari M, Mousavifar SA, Haghani H. Mobile phone text messaging and Telephone follow-up in type 2 diabetic patients for 3 months: a comparative study. Journal of Diabetes and Metabolic Disorders. 2012;11:7.

10. Zaman Huri H, Fun Wee H. Drug related problems in type 2 diabetes patients with hypertension: a cross-sectional retrospective study. BMC Endocrine Disorders. 2013;13:2.

11. McCreight LJ, Bailey CJ, Pearson ER. Metformin and the gastrointestinal tract. Diabetologia. 2016;59:426-435.

12. Riaz T, Riaz H, Hussain SA, et al. SMS reminders: future in self-care management of diabetes mellitus. Diabetology and Metabolic Syndrome. 2012;4:31. 